\title{
Age, atherosclerosis and type 2 diabetes reduce human mesenchymal stromal cell-mediated T-cell suppression
}

\author{
Ozge Kizilay Mancini ${ }^{1}$, Dominique Shum-Tim² ${ }^{2}$ Ursula Stochaj ${ }^{3}$, José A. Correa ${ }^{4}$ and Inés Colmegna ${ }^{5,6^{*}}$
}

\begin{abstract}
To this end human MSCs were isolated from adipose tissue and the MSC:CD4 ${ }^{+}$T-cell suppression was assessed in a co-culture system. In summary, this study demonstrates that advanced age, atherosclerosis and type 2 diabetes mellitus reduce the functional potency of MSCs. Optimizing the criteria for the selection of MSC donors could enhance the results of cell-based therapies.
\end{abstract}

\section{Findings}

Age and age- associated conditions (type 2 diabetes and athrosclerosis) decrease the immunomodulatory capacity of MSCs.

Optimal donor selection and proper immunological characterization of MSCs are critical for the successful therapeutic use of MSCs.

\section{Introduction}

Atherosclerotic ischemic heart disease is the leading cause of death in developed countries. The prevalence, incidence, and severity of atherosclerosis (ATH) markedly increase with chronological age and in the context of age-associated chronic inflammatory conditions such as type 2 diabetes mellitus (T2DM) [1]. Chronic inflammation is a key regulatory process that links multiple risk factors for ATH and its complications to altered arterial biology. In mature atherosclerotic lesions, immune responses mediated by $\mathrm{CD} 4^{+} \mathrm{T}$ cells seem to be critical to accelerate atherogenesis and to promote plaque instability [2]. This is supported by the correlation between increased circulating numbers of activated $\mathrm{CD} 4^{+} \mathrm{T}$ cells and the extent of ATH in carotid and coronary arteries, and by the

\footnotetext{
* Correspondence: ines.colmegna@mcgill.ca

${ }^{5}$ Division of Rheumatology, Department of Medicine, McGill University, Montreal, QC, Canada

${ }^{6}$ Royal Victoria Hospital, McGill University Health Centre, 1001 Boulevard, Décarie, Montréal, Québec H4A 3J1, Canada

Full list of author information is available at the end of the article
}

larger number of these cells in unstable plaques compared to those from patients with stable coronary artery disease $[3,4]$. In vivo studies also showed the arrest in the development and progression of ATH following $\mathrm{T}$ cell-targeted therapy (i.e., anti-CD3Ab) [5]. The fundamental role of immune-activation in ATH provides the rationale to develop therapeutic interventions that restore immune homeostasis in ischemic heart disease. Among these strategies, the use of mesenchymal stromal cells (MSCs) showed promise in preclinical studies and most recently in patients with nonrevascularizable ischemic myocardium (reviewed in $[6,7])$. Immunosuppressive and anti-inflammatory effects of MSCs are key mechanisms underlying their therapeutic effects [8]. A critical aspect linked to the success of any type of cell therapy is the appropriate selection of donors; however, the effect of donor's age and age-associated co-morbidities on human MSC-mediated T-cell suppression remains undefined $[9,10]$. The aim of this study was to evaluate the impact of chronological aging and of the age-associated diseases, ATH and T2DM, on the immunomodulatory capacity of MSCs.

\section{Methods}

The McGill University Health Center Ethics Review Board approved the study and participants provided written informed consent. Subcutaneous adipose tissue was obtained from patients undergoing programmed cardiovascular surgery. Table 1 summarizes the demographics and cardiovascular risk factors of the studied 
subjects. A full description of methods is provided as supplementary data (Additional file 1: Supplementary materials and methods). Briefly, MSCs were derived from adipose tissue and proven to meet the International Society for Cellular Therapy definition criteria [11]. Freshly harvested, early passage (P4) MSCs were used in all assays. Peripheral blood mononuclear cells (PBMCs) were obtained from a single unrelated donor, monocyte depleted ( $<5 \%$ monocytes) [12], Carboxyfluorescein succinimidyl ester (CFSE) stained and activated with CD3/CD28 beads. MSC-dependent $\mathrm{CD}_{4}^{+} \mathrm{T}$-cell suppression was assessed in co-cultures [13]. Proliferation curves of live $\mathrm{CD} 4^{+} \mathrm{T}$ cells were plotted and the suppressive effect of MSCs on $\mathrm{T}$ cells was determined by comparing maximal proliferation ( $\mathrm{T}$ cells alone) versus proliferation in co-cultures (MSCs + T cells) (Additional file 2: Figure S1). Wilcoxon's Rank Sum test was used for group comparisons. Multiple linear regression analysis examined the effects of age, ATH and T2DM on the mean MSC:CD4 ${ }^{+}$T-cell suppression capacity, after adjusting for the covariates of interest. Assumptions of the regression model were investigated with graphical analysis of residuals. All analyses were performed using SAS version 9.2 (SAS Institute Inc., Cary, NC, USA). All hypotheses tests were two-sided and performed at a significance level of 0.05 .

\section{Results}

MSCs from older donors are less efficient at suppressing T-cell proliferation

The immunomodulatory capacity of adult MSCs (AMSCs, <65 years old, $\mathrm{n}=27$ ) and elderly MSCs (E-
MSCs, $\geq 65$ years old, $n=23$ ) was examined by analyzing their ability to inhibit the proliferation of anti-CD3/ CD28-activated $\mathrm{CD}_{4}^{+} \mathrm{T}$ cells. The suppressive effect of A-MSCs and E-MSCs on CD4 ${ }^{+}$T-cell proliferation was dose-dependent. At a MSC:T cell ratio of 1:8 (Fig. 1a), A-MSCs (median $33.9 \%$, interquartile range (IQR) 6.846.0, $\mathrm{n}=27$ ) inhibited activated $\mathrm{CD}^{+} \mathrm{T}$ cells more effectively than E-MSCs (median 47.5\%, IQR 35.6-58.0, $\mathrm{n}=23)(p<0.003)$. Similar results were obtained at MSC:T cell ratios of 1:14 and 1:20. A-MSCs at 1:14 ratio (median 50.1 \% IQR 37.6-62.2, $\mathrm{n}=17$ ) had similar inhibitory capacity as E-MSCs at 1:8 ratio, highlighting the magnitude of the E-MSC defect (Fig. 1b). MSC donor age positively correlated with $\mathrm{T}$-cell proliferation in both ATH and non-ATH (samples from patients undergoing valve replacement surgery) groups (Pearson's $r=0.4$ and 0.7 , respectively) (Fig. 1c), indicating an age-associated decline in the MSC immunomodulatory capacity. Similar defects of the E-MSC suppressive ability were observed on $\mathrm{CD}^{+}{ }^{+} \mathrm{T}$ cells (Additional file 3: Figure S2). In a multiple linear regression model (Table 2), adjusting for covariates of interest, age had a significant effect on the reduction of MSC:CD4 ${ }^{+}$T-cell suppression $(p=0.02)$, with increasing mean $\mathrm{CD} 4^{+}$T-cell proliferation of $0.5 \%$ (95\% confidence interval (CI) $0.1-1.0)$ for any 1-year increase in age.

\section{ATH and T2DM reduce the immunomodulatory capacity of MSCs}

The effect of biological aging on human MSC-mediated T-cell suppression was tested by evaluating MSCs from donors with ATH and T2DM, diseases associated with

Table 1 Demographic characteristics of the study subjects

\begin{tabular}{|c|c|c|}
\hline & \multicolumn{2}{|l|}{ Surgical procedure } \\
\hline & Coronary artery by-pass & Valve replacement \\
\hline & graft (ATH) & (non-ATH) \\
\hline & $\mathrm{n}=41$ & $\mathrm{n}=9$ \\
\hline Sex (female:male, n) & $13: 28$ & $5: 4$ \\
\hline Age in years, mean (SD) & $63.4(13.3)$ & $59.7(14.8)$ \\
\hline Ethnicity (Caucasian:Asian, n) & $37: 4$ & $9: 0$ \\
\hline Body mass index, mean (SD) & $29.1(9.2)$ & $27.3(12.1)$ \\
\hline \multicolumn{3}{|l|}{ Cardiovascular risk factors, n (\%) } \\
\hline Tobacco & $20(49)$ & $4(44)$ \\
\hline Hypertension & $33(80)$ & $6(66)$ \\
\hline Hypercholesterolemia & $33(80)$ & $4(44)$ \\
\hline Type 2 diabetes & $12(29)$ & $4(44)$ \\
\hline \multicolumn{3}{|l|}{ Medications, n (\%) } \\
\hline Statins & $33(80)$ & $5(55)$ \\
\hline ACE inhibitors/ARB & $20(49)$ & $2(22)$ \\
\hline Beta blockers & $26(63)$ & $2(2)$ \\
\hline
\end{tabular}




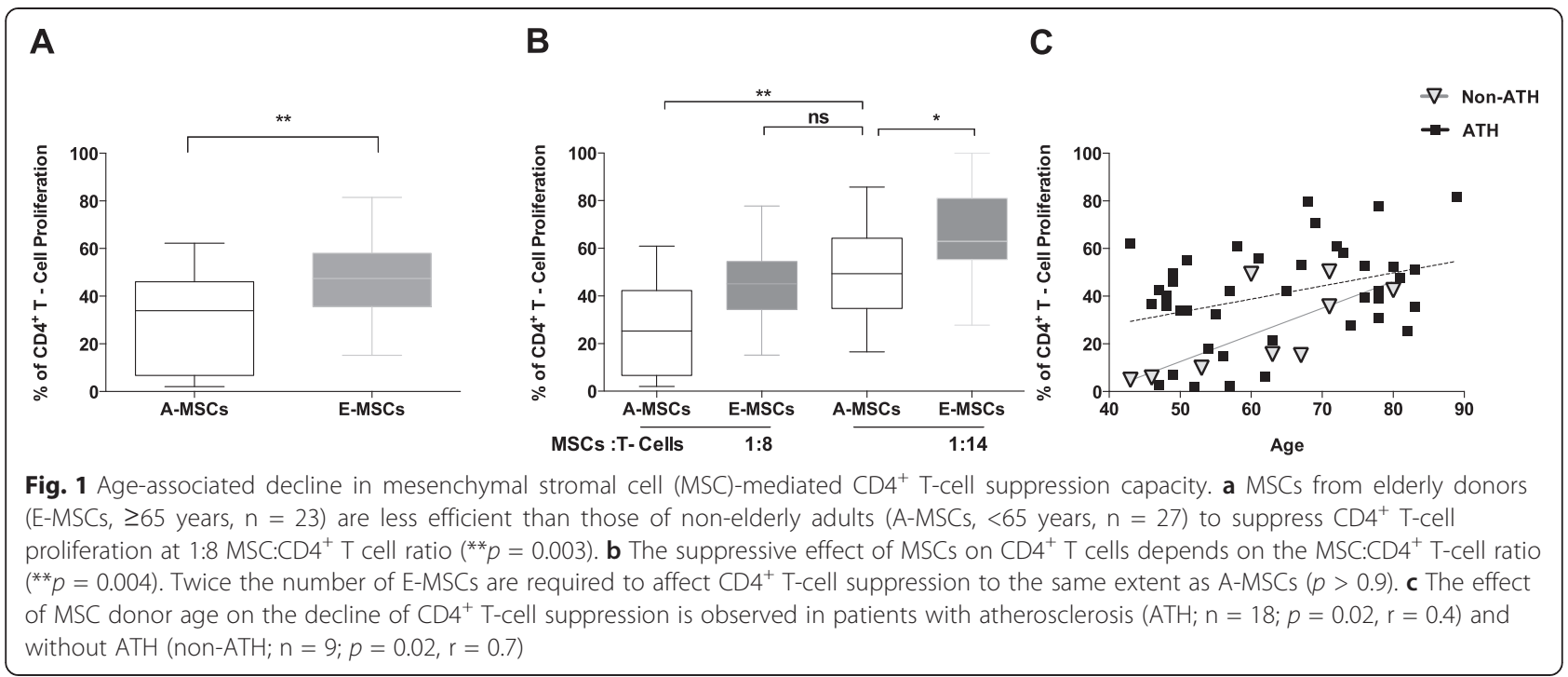

chronic inflammation and premature aging. MSCs from patients undergoing valve replacement surgery who had a normal pre-surgical coronary angiography (non-ATH subjects; median $15.6 \%$, IQR 9.9-42.5, n = 9) had a higher ability to suppress activated $\mathrm{CD} 4^{+} \mathrm{T}$ cells compared to MSCs from ATH age-matched controls (median 44.2, IQR 36.7-55.7, $\mathrm{n}=18$ ) (Fig. 2a). Moreover, in agematched ATH patients the diagnosis of T2DM further reduced the MSC suppressive capacity (median 41.0, IQR 32.3-50.1, and 56.4, IQR 40.8-74.2, for non-T2DM/ $\mathrm{T} 2 \mathrm{DM}, \mathrm{n}=12$, respectively) (Fig. 2b). Unadjusted, agematched comparisons of MSC function in a sample subset $(\mathrm{n}=7)$ suggested that ATH and T2DM were associated with a reduction in the MSC suppressive capacity (Fig. 2c). In the multiple regression model (Table 2), the presence/ absence of ATH and T2DM in MSC donors had

Table 2 Results of linear regression analysis of factors associated with mesenchymal stromal cell immunomodulatory function

\begin{tabular}{llclc}
\hline Variable & Estimate & Standard error & $T$ & $p$ value \\
\hline Intercept & -5.62 & 16.28 & -0.35 & 0.73 \\
Presence of ATH & $\mathbf{2 1 . 6 1}$ & $\mathbf{7 . 6 8}$ & $\mathbf{2 . 8 2}$ & $\mathbf{0 . 0 1}$ \\
Presence of T2DM & $\mathbf{1 4 . 3 7}$ & $\mathbf{5 . 8 0}$ & $\mathbf{2 . 4 8}$ & $\mathbf{0 . 0 2}$ \\
Age & $\mathbf{0 . 5 4}$ & $\mathbf{0 . 2 3}$ & $\mathbf{2 . 4 2}$ & $\mathbf{0 . 0 2}$ \\
Sex & 2.58 & 5.90 & 0.44 & 0.66 \\
Tobacco & -4.55 & 5.72 & -0.80 & 0.43 \\
LVD & -1.67 & 6.76 & -0.25 & 0.81 \\
Statins & -9.00 & 6.96 & -1.44 & 0.16 \\
ACE inhibitors/ARB & -4.05 & 5.72 & -0.71 & 0.48 \\
Beta blockers & -5.14 & 6.42 & -0.80 & 0.43 \\
\hline
\end{tabular}

Entries in bold are statistically significant. ACE angiotensin-converting enzyme, $A R B$ angiotensin II receptor blockers, LVD left ventricular dysfunction, T2DM type 2 diabetes mellitus significant effects on their capacity to suppress proliferating $\mathrm{CD}^{+}{ }^{+} \mathrm{T}$ cells. Subjects with $\mathrm{ATH}$ had a higher mean percentage of proliferating $\mathrm{CD}^{+} \mathrm{T}$ cells (decreased MSC:T-cell suppressive capacity) than those without ATH (mean difference $21.6 \%$; 95 \% CI, 36.137.1). Similarly, subjects with T2DM had a higher mean percentage of proliferating $\mathrm{CD} 4^{+} \mathrm{T}$-cells (mean difference $14.4 \%$; $95 \%$ CI, 2.6-26.1). There was no statistically significant interaction either between ATH and T2DM or between each of them and age. This suggests that the effect of ATH is independent of T2DM and that these effects are also independent of age.

\section{Discussion}

The PRECISE trial demonstrated the feasibility, safety and potential therapeutic benefit of the transendocardial administration of autologous adipose-derived MSCs in no-option patients with ischemic cardiomyopathy [7]. This study provided a proof of concept to test MSCs in larger ongoing clinical trials [6]. However, the improvement of cardiac function varied in preclinical trials. This emphasizes the need to define determinants of MSC therapeutic efficiency that will inform on proper donor selection [7, 14]. Previous data suggest that the aging process may impair the functional activity of murine MSCs, limiting their therapeutic potential [15]. Delineating the effects of aging and age-associated conditions on MSC function is critical, since the vast majority of patients that would benefit from MSC use in the context of ATH are elderly individuals. Notably, a significant proportion of this cohort also has T2DM [16]. Furthermore, defining the factors that impact MSC function would help to identify proper criteria to select the most therapeutically efficient cells for clinical applications. 


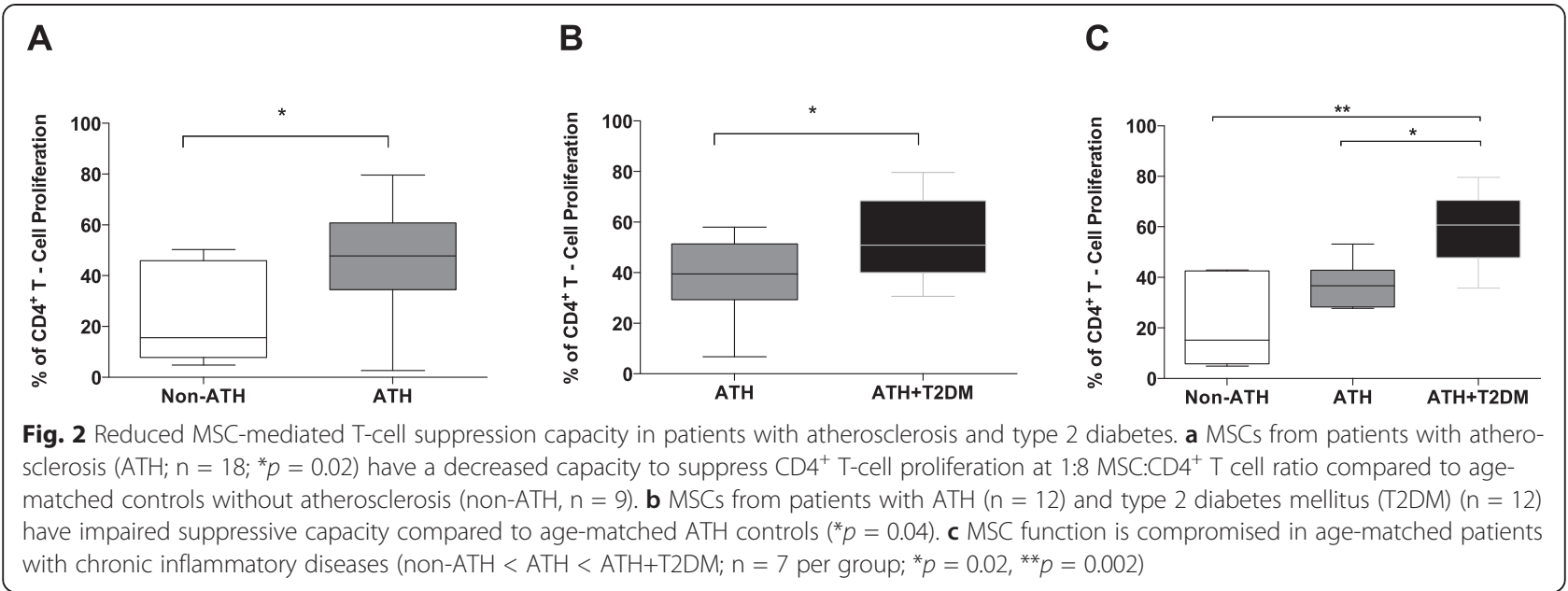

Recent evidence confirmed that early-passage (i.e., passages 2-4) and freshly harvested MSCs have better in vitro T-cell suppression capacity and are associated with more clinical benefits than late-passage and freeze/ thawed MSCs [17]. Our findings suggest that even when tested under "optimal" conditions (i.e., fresh early-passage MSCs in a reproducible immunopotency assay [13]), MSCs from elderly subjects with ATH have impaired T-cell suppression compared to their non-elderly adult counterparts. Consistent with previous studies [18, 19], this impaired function was not explained by differences in the MSC proliferative capacity or phenotype. Similar to E-MSCs, MSCs from donors with ATH and T2DM have reduced MSCmediated T-cell suppression, and the coexistence of these chronic inflammatory conditions further compromise MSC function.

The overall efficacy of stem cell transplantation relies on the activity of donor cells and tissue environment. Our co-culture model is limited to MSCs and PBMCs and does not fully simulate all the components of the in vivo ischemic and inflammatory environment. However, this system predicts the MSC immunomodulatory potency, which is the most relevant mechanism for the therapeutic effect of MSCs [13]. The finding that age, T2DM and ATH are associated with reduced MSC immunomodulatory function is in line with a recent report suggesting that MSCs from subjects over 60 years of age have a reduced ability to ameliorate myocardial function compared to patients younger than 40 years [20]. A reduction of MSC-secreted angiogenic factors [18], an increased vulnerability to hypoxic injury [21] and higher levels of miR-335 are mechanisms proposed to account for the diminished reparative activities of E-MSCs.

\section{Conclusions}

In summary, our results indicate that age and ageassociated conditions (T2DM and $\mathrm{ATH}$ ) decrease the immunomodulatory capacity of MSCs, highlighting the relevance of donor selection and the need for proper immunological characterization of MSCs. Understanding the interplay between aging, MSC function and their clinical implications remains the only rational path to the successful therapeutic use of MSCs.

\section{Additional files}

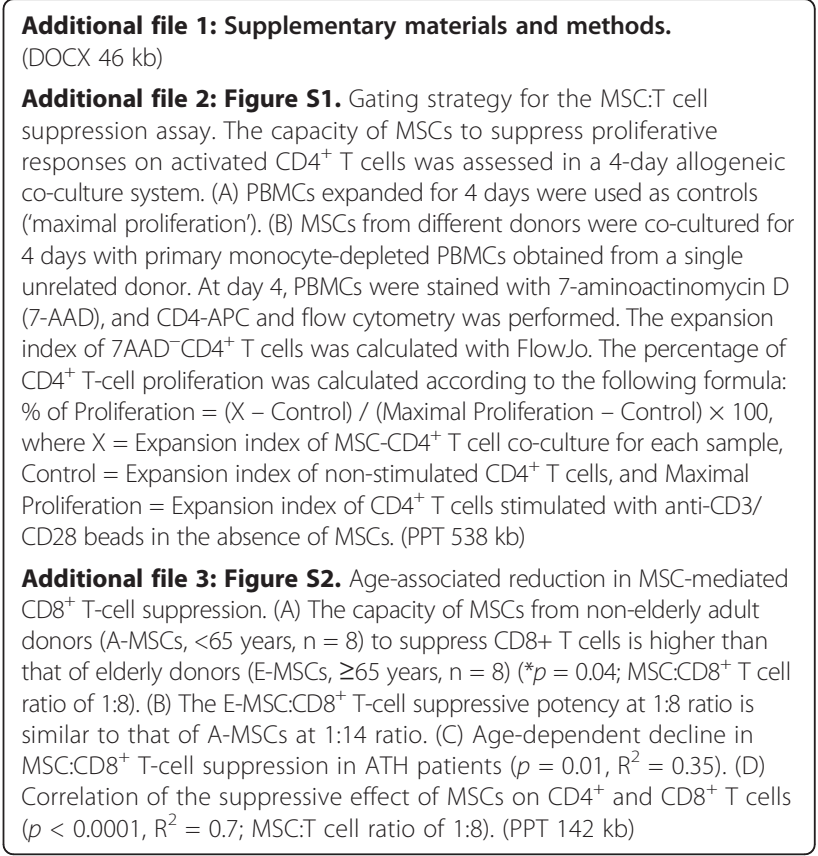

\section{Abbreviations}

A-MSC: Adult mesenchymal stromal cell; ATH: Atherosclerosis; Cl: Confidence interval; E-MSC: Elderly mesenchymal stromal cell; IQR: Interquartile range; MSC: Mesenchymal stromal cell; non-ATH: Non-atherosclerosis (samples from patients undergoing valve replacement surgery); PBMC: Peripheral blood mononuclear cell; T2DM: Type 2 diabetes mellitus. 


\section{Competing interests}

The authors declare that they have no competing interests.

\section{Authors' contributions}

OKM carried out the experiments, analyzed and interpreted the data, and wrote the first manuscript draft. DST collected all human MSC samples. US assisted in data interpretation. JAC performed the statistical analysis, contributed to data interpretation and provided all statistical descriptions for the manuscript. IC designed and coordinated the study, assisted in data interpretation, reviewed the manuscript critically, and has given final approval of the version to be published. All authors read and approved the final manuscript.

\section{Acknowledgements}

This work was supported by an operating grant from the Canadian Institutes of Health Research (CIHR) and the Programme de bourses de Chercheur-boursier clinicien from the Fonds De Recherche Sante Quebec. The authors are grateful to Maximilien Lora for his help in optimizing the conditions of the initial experiments.

\section{Author details}

'Department of Anatomy and Cell Biology, McGill University, Montreal, QC, Canada. ${ }^{2}$ Division of Cardiothoracic Surgery and Surgical Research, Royal Victoria Hospital, McGill University Health Center, Montreal, QC, Canada. ${ }^{3}$ Department of Physiology, McGill University, Montreal, QC, Canada. ${ }^{4}$ Department of Mathematics and Statistics, McGill University, Montreal, QC, Canada. ${ }^{5}$ Division of Rheumatology, Department of Medicine, McGill University, Montreal, QC, Canada. ${ }^{6}$ Royal Victoria Hospital, McGill University Health Centre, 1001 Boulevard, Décarie, Montréal, Québec H4A 3J1, Canada.

Received: 10 March 2015 Revised: 12 May 2015

Accepted: 8 July 2015 Published online: 08 August 2015

\section{References}

1. D'Agostino Sr RB, Pencina MJ, Massaro JM, Coady S. Cardiovascular disease risk assessment: insights from Framingham. Global Heart. 2013;8:11-23. doi:10.1016/j.gheart.2013.01.001.

2. Hedrick CC. Lymphocytes in atherosclerosis. Arterioscler Thromb Vasc Biol. 2015;35:253-7. doi:10.1161/ATVBAHA.114.305144.

3. Ammirati $E$, Cianflone D, Vecchio V, Banfi M, Vermi AC, De Metrio M, et al. Effector memory $T$ cells are associated with atherosclerosis in humans and animal models. J Am Heart Assoc. 2012;1:27-41. doi:10.1161/JAHA.111.000125.

4. De Palma R, Del Galdo F, Abbate G, Chiariello M, Calabro R, Forte L, et al. Patients with acute coronary syndrome show oligoclonal T-cell recruitment within unstable plaque: evidence for a local, intracoronary immunologic mechanism Circulation. 2006;113:640-6. doi:10.1161/CIRCULATIONAHA.105.537712.

5. Kita T, Yamashita T, Sasaki N, Kasahara K, Sasaki Y, Yodoi K, et al. Regression of atherosclerosis with anti-CD3 antibody via augmenting a regulatory T-cell response in mice. Cardiovasc Res. 2014;102:107-17. doi:10.1093/cvr/cvu002.

6. Karantalis V, Hare JM. Use of mesenchymal stem cells for therapy of cardiac disease. Circ Res. 2015;116:1413-30. doi:10.1161/CIRCRESAHA.116.303614.

7. Perin EC, Sanz-Ruiz R, Sanchez PL, Lasso J, Perez-Cano R, Alonso-Farto JC, et al. Adipose-derived regenerative cells in patients with ischemic cardiomyopathy: the PRECISE Trial. Am Heart J. 2014;168:88-95. doi:10.1016/j.ahj.2014.03.022.e2.

8. Ranganath SH, Levy O, Inamdar MS, Karp JM. Harnessing the mesenchymal stem cell secretome for the treatment of cardiovascular disease. Cell Stem Cell. 2012;10:244-58. doi:10.1016/j.stem.2012.02.005.

9. Li XY, Ding J, Zheng ZH, Li XY, Wu ZB, Zhu P. Long-term culture in vitro impairs the immunosuppressive activity of mesenchymal stem cells on $T$ cells. Mol Med Rep. 2012;6:1183-9. doi:10.3892/mmr.2012.1039.

10. Landgraf K, Brunauer R, Lepperdinger G, Grubeck-Loebenstein B. The suppressive effect of mesenchymal stromal cells on T cell proliferation is conserved in old age. Transpl Immunol. 2011;25:167-72. doi:10.1016/j.trim.2011.06.007.

11. Dominici M, Le Blanc K, Mueller I, Slaper-Cortenbach I, Marini F, Krause D, et al. Minimal criteria for defining multipotent mesenchymal stromal cells. The International Society for Cellular Therapy position statement. Cytotherapy. 2006:8:315-7. doi:10.1080/14653240600855905.

12. Barrett L, Dai C, Gamberg J, Gallant M, Grant M. Circulating CD14-CD36+ peripheral blood mononuclear cells constitutively produce interleukin-10. J Leukoc Biol. 2007;82:152-60. doi:10.1189/jlb.0806521.
13. Bloom DD, Centanni JM, Bhatia N, Emler CA, Drier D, Leverson GE, et al. A reproducible immunopotency assay to measure mesenchymal stromal cell-mediated T-cell suppression. Cytotherapy. 2015;17:140-51. doi:10.1016/j.jcyt.2014.10.002.

14. Houtgraaf JH, den Dekker WK, van Dalen BM, Springeling $T$, de Jong $R$, van Geuns RJ, et al. First experience in humans using adipose tissuederived regenerative cells in the treatment of patients with ST-segment elevation myocardial infarction. J Am Coll Cardiol. 2012;59:539-40. doi:10.1016/j.jacc.2011.09.065.

15. Liang H, Hou H, Yi W, Yang G, Gu C, Lau WB, et al. Increased expression of pigment epithelium-derived factor in aged mesenchymal stem cells impairs their therapeutic efficacy for attenuating myocardial infarction injury. Eur Heart J. 2013;34:1681-90. doi:10.1093/eurheartj/ehr131.

16. Booth GL, Kapral MK, Fung K, Tu JV. Relation between age and cardiovascular disease in men and women with diabetes compared with non-diabetic people: a population-based retrospective cohort study. Lancet. 2006;368:29-36. doi:10.1016/S0140-6736(06)68967-8.

17. Moll G, Alm JJ, Davies LC, von Bahr L, Heldring N, Stenbeck-Funke L, et al. Do cryopreserved mesenchymal stromal cells display impaired immunomodulatory and therapeutic properties? Stem cells. 2014. doi:10.1002/stem.1729.

18. Efimenko A, Dzhoyashvili N, Kalinina N, Kochegura T, Akchurin R, Tkachuk V, et al. Adipose-derived mesenchymal stromal cells from aged patients with coronary artery disease keep mesenchymal stromal cell properties but exhibit characteristics of aging and have impaired angiogenic potential. Stem Cells Transl Med. 2014;3:32-41. doi:10.5966/sctm.2013-0014.

19. Haack-Sorensen M, Friis T, Mathiasen AB, Jorgensen $E$, Hansen L, Dickmeiss $E$, et al. Direct intramyocardial mesenchymal stromal cell injections in patients with severe refractory angina: one-year follow-up. Cell Transplant. 2013;22:521-8. doi:10.3727/096368912X636830.

20. Liu Y, Liu T, Han J, Yang Z, Xue $X$, Jiang H, et al. Advanced age impairs cardioprotective function of mesenchymal stem cell transplantation from patients to myocardially infarcted rats. Cardiology. 2014;128:209-19. doi:10.1159/000360393.

21. Zhang Z, Zhao C, Liu B, Liang D, Qin X, Li X, et al. Inositol pyrophosphates mediate the effects of aging on bone marrow mesenchymal stem cells by inhibiting Akt signaling. Stem Cell Res Ther. 2014;5:33. doi:10.1186/scrt431.

\section{Submit your next manuscript to BioMed Central and take full advantage of:}

- Convenient online submission

- Thorough peer review

- No space constraints or color figure charges

- Immediate publication on acceptance

- Inclusion in PubMed, CAS, Scopus and Google Scholar

- Research which is freely available for redistribution 